EPJ Web of Conferences 71, 00056 (2014)

DOI: $10.1051 /$ epjconf / 20147100056

(C) Owned by the authors, published by EDP Sciences, 2014

\title{
Doubly strange system physics with antiprotons at PANDA
}

\author{
Felice Iazzi ${ }^{1 \mathrm{a}, 2}$ \\ (on behalf of PANDA Collaboration) \\ ${ }^{1}$ Dept. of Applied Science and Technology, Politecnico di Torino, Torino, Italy \\ ${ }^{2}$ INFN-Sezione di Torino, Torino, Italy
}

\begin{abstract}
The study of the doubly strange hyper-systems represents a step forward in understanding the unexplored world of the strange matter in the frame of a better knowledge of the hyperon-nucleon and hyperon-nucleus interaction. The production of double hyper-systems, up to now, have been based on the use of kaon beams through a double strangeness exchange reaction. A new technique has been designed by the PANDA Collaboration, which will use the antiprotons at $3 \mathrm{GeV} / \mathrm{c}$ of the HESR facility at FAIR to create doubly strange hyperons and drive them into nuclear targets. This technique requires the use of 2 targets, located inside and outside the beam pipe. In spite of the constraints arising from the presence of a solid target inside an antiproton ring, the technique looks promising in terms of rate of hyperons and hyper-nuclei produced. After a review of the physics items that will be investigated in the hyper-nuclear section of PANDA experiment, the characteristics of the antiprotons facility, the results of the feasibility study of the 2-target technique, the design of the hyper-nuclear set-up in PANDA and the expected rates of the double hyper-nuclei will be presented.
\end{abstract}

\section{Introduction}

Recently the interest for the doubly strange systems rose up in the community of the strangeness physics. The reasons are twofold: on one side the $S=-2$ systems allow to get a step forward in understanding the interaction of the strange matter with nucleons and nuclei, on the other side the availability of intense beams at the new hadronic machines (JPARC, FAIR) allows a copious production of doubly strange hyperons.

There are 3 kinds of doubly strange systems: the exotic $\Xi^{-}$-atoms, the $\Xi^{-}$hyper-nuclei and the $\Lambda \Lambda$ hyper-nuclei (also called Double Hyper-nuclei, $D H$ ). In these systems the hyperons interact with the nucleus, with the nucleons and with each other respectively, as described in the Section 2. In spite of the great interest for these interactions, the amount of data remained very scarce till today, due to the difficulty of producing such systems. On the other hand, the alternative way to investigate the effects of the double strangeness contents on the hyperon potentials is to perform scattering experiments, but the short lifetime of both $\Xi^{-}$and $\Lambda$ hyperons do not allow to have these hyperons as projectiles. For these reasons, after the first claim of the existence of the Double Hyper-nuclei in 1963 by Danysz and Pniewsky [1], the search for the doubly strange systems didn't produce data till the 90 -ties of the past

\footnotetext{
${ }^{\mathrm{a}}$ Corresponding author: felice.iazzi@polito.it
}

This is an Open Access article distributed under the terms of the Creative Commons Attribution License 2.0, which permits unrestricted use, distribution, and reproduction in any medium, provided the original work is properly cited. 
century, when the availability of intense kaon beams restarted a number of experiments at KEK and AGS. The results of these experiments are reported in the next Sections.

Nevertheless the statistics obtained in these experiments is still quite poor: the number of observed $\mathrm{S}=-2$ systems was of the order of few tens. Moreover the data were spread among different hypersystems. This allowed to confirm the existence of $\Xi^{-}$and $\Lambda \Lambda$ hyper-nuclei (no $\Xi^{-}$-atom has been observed up to now) but was insufficient to obtain a reliable information about the $\Xi^{-}$hyper-nuclear levels and the $\Lambda \Lambda$ separation energy.

A big step forward seems to be in sight with the advent of the new hadronic machines FAIR and JPARC, which will supply very intense beams of antiprotons and kaons. The physics program of both facilities include experiments dedicated to the $\mathrm{S}=-2$ hyper-systems (PANDA at FAIR [2] and E03+E07 at JPARC [3]). A considerable statistics is expected not only because of the beam intensity but also from the improvement of the production and detection techniques. In particular the totally new technique designed in PANDA, which will use antiprotons instead of kaons, will be illustrated in Sections 3, 4, 5 and 6, together with the expected statistics and the results of the tests on parts of the detector. The conclusions will be drawn in Section 7.

\section{The physics of the $S=-2$ systems}

The peculiar physics that can be investigated in forming systems with $\mathrm{S}=-2$ concerns: a) the doublystrange hyperon-nucleus ( $\Xi^{-}$-nucleus) interaction, b) the doubly-strange hyperon-nucleon $\left(\Xi^{-}-\mathrm{N}\right)$ interaction, $\mathrm{c})$ the hyperon-hyperon $(\Lambda-\Lambda)$ interaction and d) the hyperon induced non mesonic weak decay $(\Lambda \Lambda \rightarrow \Lambda N)$. The two latter items can only be studied in $\Lambda \Lambda$-hypernuclei. The $X$-ray spectra of the $\Xi^{-}$cascade in the hyperonic atom, the $\gamma$ emission in the $\Xi^{-}$- nuclear capture, the binding energy excess of the $\Lambda \Lambda$-hyper-nuclear system and the emitted pion energy in the $\Lambda \Lambda$ decay are the relevant measurements to be performed in the experiments.

\subsection{Exotic $\Xi^{-}$atoms}

The exotic $\Xi^{-}$atom contains a $\Xi^{-}$hyperon in one of the atomic levels. This type of exotic atoms belongs to the class of the hadronic atoms, which includes the mesonic atoms ( $\pi^{-}$and $\mathrm{K}^{-}$atoms), the anti-protonic atoms and the baryonic atoms $\left(\Sigma^{-}, \Xi^{-}\right.$and $\Omega^{-}$atoms $)$. These atoms are sensitive to both electromagnetic and strong forces and this peculiarity makes them ideal systems to study the interplay of both interactions. The most striking results were obtained from the $X$ ray emission during the cascade of the hadron from the high to the low levels. The masses of $\pi^{-}$[4] and $K^{-}$[5] were measured with high precision in mesonic atoms and important results have been obtained from the accurate measurements of the antiproton mass measured in anti-protonic atoms and atom $/ \mathrm{molecules,}$, as reported in [6]. Taking into account that the masses of the strange negative baryons are greater than those ones of the mesons and antiprotons and their atomic levels have smaller radii, the same level of accuracy in their measurements (in the hydrogen-like approximation) is expected to be achievable.

Another important information can be obtained concerning the interaction of the strange baryons with the nucleus. The hadronic atoms are formed after capture of a negative hadron in a high level $n_{c} \approx n_{e} \cdot \sqrt{M_{h} / m_{e}}$ ( $n_{e}$ being the highest occupied level of the atom, $M_{h}$ and $m_{e}$ are the hadron and electron masses respectively). The hadron decays into lower levels and this atomic cascade leads the 
hyperon into a level, where the wave function overlaps the peripheral region of the nucleus. Here the hadron is sensible not only to the Coulomb potential but also to the nuclear forces, which shift the atomic levels and broaden their widths. The measured values of shift and width can be used to test models of the hyperon-nucleus interaction in the nuclear periphery. It must be noticed that the broadening effect of the nuclear force is the rapid absorption inside the nucleus. Therefore only one atomic transition, from the last atomic level to the first broadened one $n_{a b s}$ (called absorption level), can be observed. Moreover, it has been suggested [7] that the well depth could depend on the atomic mass number $A$. Therefore, higher is the number of investigated hyperons and atoms, better is the knowledge of the strangeness role in the interaction with nuclei.

The available data concerning the exotic atoms cover practically the whole periodic table but only the $\Sigma^{-}$is present among the hyperons. This situation could improve quite a lot in the next experiments at JPARC and FAIR.

\section{$2.2 \Xi^{-}$hypernuclei}

The absorption of one $\Xi^{-}$inside a nucleus creates a hyper-nucleus with atomic number decreased by 1 and baryon number increased by 1 with respect to the original nucleus:

$$
\Xi^{-}+{ }^{Z} A \rightarrow{ }_{\Xi}^{Z-1} A+\gamma
$$

Spectroscopy measurements can give information about the levels of the nuclear field. It must be remarked that the hyperon transitions through the nuclear levels can be suppressed by the competition with the $Y N \rightarrow Y N$ (scattering and charge exchange) and $\Xi^{-} p \rightarrow \Lambda \Lambda$ (strangeness transfer) interactions, depending on the transition $\left(\Gamma_{\Xi}\right)$ and reaction $\left(\Gamma_{Y N-Y N, Y Y}\right)$ widths. There are calculations that estimate $\Gamma_{\Xi p->\Lambda \Lambda}$ not too larger $(\approx 5 \mathrm{MeV})$ than $\Gamma_{\Xi}$ (typically of the order of $1 \mathrm{MeV}$ for $s$ and $p$ states, see for example $[8,9,10])$. Future experiments should provide a very high statistics to measure the levels and confirm or reject these estimations of the widths.

Inside the nucleus the hyperon interacts strongly with nucleons. In the short range interaction the $s$ quarks come into play, while in the long range the boson exchange plays the main role. There are some peculiar aspects [11] of the OBE picture: in the $Y N \rightarrow Y N$ interaction only the non-strange mesons of isospin $I=0,1(\pi, \eta, \omega, \rho \ldots)$ can be exchanged, while in the $\Xi^{-} p \rightarrow \Lambda \Lambda$ interaction only strange mesons of isospin $I=1 / 2$ can be exchanged.

Another very interesting interaction of the $\Xi^{-}$hyperon inside the nucleus is the Non Mesonic Weak Decay (NMWD), which, analogously to that one of the $\Lambda$, proceeds via:

$$
\Xi^{-}+p \rightarrow \Lambda+n ; \quad \Xi^{-}+p \rightarrow \Sigma^{-}+p ; \text { and } \quad \Xi^{-}+n \rightarrow \Sigma^{-}+n
$$

These decays involve the weak interaction and they are expected to be strongly suppressed by the strong interaction processes discussed above. Therefore the statistics required to obtain even few data seems out of the possibilities of the upcoming experiments. Nevertheless, results about NMWD of $\Xi^{-}$should be welcome, because they might shed light on the behaviour of the strangeness in the

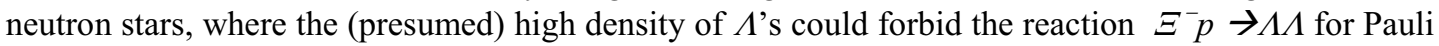
blocking. 
The status of art of the $\Xi^{-}$-hyperon is quite poor. The binding energy $B_{\Xi}$ of few hyper-nuclei, measured at Berkely and CERN, are reported in Table 1:

Table 1 Measured Binding Energy $\left(B_{\Xi}\right)$ of some $\Xi^{-}$hyper-nuclei (in squared brackets: the references)

\begin{tabular}{|l|l|l|l|l|l|l|l|}
\hline Hyper-nucleus & ${ }_{\Xi}^{8} \mathrm{He}[12]$ & ${ }_{\Xi}^{11} B[13]$ & ${ }_{\Xi}^{13} C[14]$ & ${ }_{\Xi}^{15} C[15]$ & ${ }_{\Xi}^{17} O[15]$ & ${ }_{\Xi}^{28} A l[15]$ & $\left.\begin{array}{r}29 / 30 \\
\Xi\end{array}\right][15]$ \\
\hline$B_{\Xi}[\mathrm{MeV}]$ & $5.9 \pm 1.2$ & $9.2 \pm 2.2$ & $18.1 \pm 3.2$ & $16 \pm 4.7$ & $16 \pm 5.5$ & $23.2 \pm 6.8$ & $2.4 \pm 6.3$ \\
\hline
\end{tabular}

From the above data an attractive potential $V_{\Xi} \approx 21 \div 24 \quad[\mathrm{MeV}]$ has been deduced [16], while other experiments at AGS (E885) and KEK (E224) produced smaller values, 14 and 16 [MeV] respectively. Other data are concerning: a) the upper limit $(28 \mathrm{mb})$ of the elastic cross section of $\Xi^{-}$ $+N$, obtained from $\Xi^{-}$produced in $\left(\mathrm{K}^{-}, \mathrm{K}^{+}\right)$reaction at $\left.1.66 \mathrm{GeV} / \mathrm{c}[17], \mathrm{b}\right)$ the mean free path $(\approx 4.7$ $[\mathrm{fm}]$ ) of $\Xi^{-}$in nucleus (at $\Xi^{-}$momentum of $\approx 0.6 \mathrm{GeV} / \mathrm{c}$ ) [18], c) the forward $\left(\theta_{\pi}<8^{\circ}\right)$ cross section $(\approx 89[\mathrm{nb} / \mathrm{sr}])$ of the reaction $\left.K^{-}+{ }^{12} C \rightarrow K^{+}+{ }^{12} B_{\Xi}[19], \mathrm{d}\right)$ the cross section $(\approx 5[\mathrm{mb}])$ of the conversion reaction $\Xi^{-}+p \rightarrow \Lambda+\Lambda$ (at $\Xi^{-}$momentum of $\approx 0.5 \mathrm{GeV} / \mathrm{c}$ ) [19].

\section{$2.3 \Lambda \Lambda$ hypernuclei}

The presence of a $\Lambda \Lambda$-pair inside the same( hyper)-nucleus makes possible their interaction. The strong interaction between both hyperons involves the $s$-quarks at short distances $(\leq 0.5 \mathrm{fm})$ and the meson exchange at large distances. As for the $Y N$ interaction, there are constraints in this case too: in the OBE picture, only non-strange mesons with null isospin $(\omega, \eta \ldots)$ can be exchanged. After formation of the double hyper-nucleus, both $\Lambda^{\text {'s }}$ can decay weakly. In addition to the mesonic and non mesonic decay of the hyperons independently on each other, there is also the possibility of a peculiar non-mesonic decay, which involves the $\Lambda-\Lambda$ weak interaction. Let's see in some detail these aspects in the next Sub-Sections.

\subsection{1 $\Lambda-\Lambda$ strong interaction}

When a $\Xi^{-}$-hyperon interacts strongly with a proton inside a $\Xi^{-}$-hyper-nucleus ${ }_{\Xi}^{Z-1} A$, can form $2 \Lambda^{\prime}$ s or a $\Xi^{0}-n$ pair, with some excess of energy:

$$
\begin{gathered}
\Xi^{-}+p \rightarrow \Lambda \Lambda \text {-pair inside the same( hyper)-nucleus } \\
\Xi^{-}+p \rightarrow \Xi^{0}+n+5[\mathrm{MeV}]
\end{gathered}
$$

Reaction 4) contains a neutron in the final state and the excess of energy is quite small. Therefore one could expect to be suppressed by Pauli blocking, especially in heavy nuclei. In the following we shall discuss only the more probable case of 2 hyperons inside the nucleus. 
The excess of energy in reaction 3) could produce $\gamma$ s or be spent in breakup of the nucleus, as argued in [20] for an $\alpha$-cluster. The residual energy of the $\Lambda$ 's in this case might be small enough to allow them to stick to the residual nucleus or to the fragments. If both $\Lambda$ 's stick to the same fragment ${ }^{Z} A$, a double hyper-nucleus $(D H)$ is formed. Inside the $D H$, each $\Lambda$ occupies a level of the nuclear potential and is bound in the nuclear field with a binding energy $B_{\Lambda}\left({ }_{\Lambda}^{Z} A\right)$. The interaction between both $\Lambda$ 's is taken into account by a further energy term, called separation energy $\Delta B_{\Lambda \Lambda}$, related to the binding energy $B_{\Lambda \Lambda}\left({ }_{\Lambda \Lambda}^{Z} A\right)$ of the 2 hyperons in the nuclear field and to the binding energy $B_{\Lambda}\left({ }_{\Lambda}^{Z} A\right)$ of each $\Lambda$ inside the single hyper-nucleus ${ }_{\Lambda}^{Z} A$ by:

$$
\Delta B_{\Lambda \Lambda}=B_{\Lambda \Lambda}\left({ }_{\Lambda \Lambda}^{Z} A\right)-2 B_{\Lambda}\left({ }_{\Lambda}^{Z} A\right)
$$

Negative or positive values of $\Delta B_{\Lambda \Lambda}$ correspond to attractive or repulsive $\Lambda \Lambda$ potentials respectively. They are obtained from Eq. 5) by measuring the binding energy $B_{\Lambda \Lambda}\left({ }_{\Lambda \Lambda}^{Z} A\right)$ of a $D H$ and comparing it to the binding energy $B_{\Lambda}\left({ }_{\Lambda}^{Z} A\right)$ of the corresponding single hyper-nucleus (single hypernuclei have been widely explored and a lot of information is present in literature). The measurement of the separation energy can help to test different potential models. Moreover, a decreasing trend with respect to increasing mass number $A$ is expected, as an effect of the smaller overlapping of the hyperons wave functions. Also interesting is the dependence of $B_{\Lambda \Lambda}\left({ }_{\Lambda \Lambda}^{Z} A\right)$ on $A$.

The available data are at present very scarce: they consist of few double hyper-nuclei, whose binding energies and separation energies have been measured, as reported in Table 2:

Table 2 Binding and separation energy of the observed $D H$ s (in squared brackets: the references)

\begin{tabular}{|c|c|c|c|c|c|c|c|}
\hline $\begin{array}{c}\text { Double } \\
\text { Hypernucleus }\end{array}$ & $\begin{array}{c}{ }_{\Lambda \Lambda}^{6} \mathrm{He} \\
{[21,23]}\end{array}$ & $\begin{array}{c}{ }_{\Lambda}{ }^{6} \mathrm{He} \\
\mathrm{Or} \\
{ }^{11} \mathrm{Be} \\
{[23]}\end{array}$ & $\begin{array}{c}{ }_{\Lambda \Lambda}^{10} B e \\
{[1,22,23]}\end{array}$ & $\begin{array}{c}{ }_{\Lambda \Lambda}^{10} \mathrm{Be} \\
\mathrm{Or} \\
{ }^{13} \mathrm{~B} \\
{ }^{13} \mathrm{C}\end{array}$ & $\begin{array}{c}{ }_{\Lambda \Lambda}^{10} B e \\
{[23]}\end{array}$ & $\begin{array}{c}{ }_{\Lambda \Lambda}^{11} B e \\
\text { Or } \\
{ }_{\Lambda \Lambda}^{12} B e \\
{[23]}\end{array}$ & $\begin{array}{l}{ }_{\Lambda \Lambda} B \\
{[23]}\end{array}$ \\
\hline$B_{\Lambda \Lambda}[\mathrm{MeV}]$ & $6.9 \pm 0.16$ & $\begin{array}{c}10.01 \pm 0.71 \\
\text { or } \\
22.12 \pm 2.67\end{array}$ & $14.7 \pm 0.4$ & $\begin{array}{c}8.5 \pm 0.7 \\
\quad \text { or } \\
27.6 \pm 0.7\end{array}$ & $11.9 \pm 0.13$ & $\begin{array}{c}20.83 \pm 2.61 \\
\text { or } \\
20.49 \pm 1.21\end{array}$ & $23.6 \pm 0.7$ \\
\hline$\Delta B_{\Lambda \Lambda}[\mathrm{MeV}]$ & $0.67 \pm 0.17$ & $\begin{array}{c}3.77 \pm 1.71 \\
\text { or } \\
3.94 \pm 2.71\end{array}$ & $1.3 \pm 0.4$ & & $-1.52 \pm 0.15$ & $2.26 \pm 1.34$ & $0.6 \pm 0.8$ \\
\hline
\end{tabular}

The data from Ref. [23] are obtained under the assumption that the $\Xi^{-}$hyperon is absorbed inside the nucleus from the atomic level 3D. Some of these data are still under analysis and could be slightly modified, other ones have been re-analysed after the fist claim. Another claimed $D H$ event [25] has not been reported in Table 2 because it was submitted to severe criticisms.

The above data set presents 3 serious problems: a) some events cannot be unambiguously assigned to one hyper-nucleus, b) data concerning the same hyper-nucleus are in disagreement out of the error bars, c) the statistics is absolutely poor. Therefore the questions about the attractive/repulsive 
potential and the dependence of the binding and separation energy on the mass number cannot yet be answered. On the other hand the existence of double hyper-nuclei seems established.

The $\Lambda \Lambda$-hyper-nuclei allow to investigate the $H$ particle, a neutral di-baryon made of 6 quarks (uuddss, $J=0, I=0$ ), predicted by Jaffe [26] in 1977. More recent calculations in LQCD found a bound $H$ di-baryon $[27,28,29]$. The existence of this particle is related to the $D H$ formation. In fact, if the $H$ mass is less than twice the $\Lambda$ mass, it is stable against the strong decay. Inside the $\Xi^{-}$hyper-nucleus the conversion reaction could produce an $H$ particle with mass $m_{H}=2 m_{\Lambda}+B_{H}$, where $B_{H}$ is the binding energy of $2 \Lambda$ 's inside $H$. Calling $B_{\Lambda \Lambda}$ the binding energy of $2 \Lambda$ 's inside the $D H$, from the energy conservation it comes that $H$ should be ejected out of the nucleus if its mass satisfies the relationship:

$$
B_{H}<2 B_{\Lambda}\left({ }_{\Lambda}^{Z} A\right)+\Delta B_{\Lambda \Lambda} \Rightarrow m_{H}<2 m_{\Lambda}+B_{\Lambda \Lambda}
$$

In this case no weak decay of $\Lambda^{\text {'s }} \mathrm{s}$ can be observed. In the other case, when Eq. 6) is not satisfied, the $H$ di-baryon should have a mass greater than the mass of both $\Lambda$ 's bound inside the hyper-nucleus. Therefore, measuring the ground state of a double hyper-nucleus, one can obtain a lower limit of the $H$ mass. Up to now, no $H$ candidates have been observed: a lower limit of $2.204[\mathrm{GeV} / \mathrm{c}]$ for the mass [30] and of $10[\mathrm{nb} / \mathrm{sr}]$ for the differential cross section of the direct production of a $\Lambda \Lambda$ hyper-nucleus by $K$ [31]. A list of the unsuccessful attempts to observe the $H$ di-baryon can be found in [32].

\subsection{2 $\Lambda-\Lambda$ weak decay}

Each $\Lambda$ hyperon inside a $D H$ undergoes mesonic (MWD) or non mesonic (NMWD) weak decay, each going into one neutral or charged meson or nucleon, as:

$$
{ }_{\Lambda \Lambda}^{A} Z \rightarrow\left\{\begin{array}{l}
{ }_{\Lambda}^{A} Z+\pi^{0} \\
{ }_{\Lambda}^{A}(Z+1)+\pi^{-}
\end{array} \quad(\mathrm{MWD}), \quad{ }_{\Lambda \Lambda}^{A} Z \rightarrow\left\{\begin{array}{l}
{ }_{\Lambda}^{A-2} Z+n+n \\
{ }_{\Lambda}^{A-2}(Z-1)+n+p
\end{array}{ }_{\Lambda}\right. \text { (NMWD) }\right.
$$

The puzzle of the discrepancy between the experimental values of the ratio $\Gamma_{n} / \Gamma_{p}$ of the widths of the neutron to proton channels in NMWD of single hyper-nuclei could be investigated also in double hyper-nuclei. Much more interesting is the study of the $\Lambda \Lambda$ weak interaction through the socalled Hyperon Induced Non Mesonic Weak Decay (HINMWD):

$$
{ }_{\Lambda \Lambda}^{A} Z \rightarrow\left\{\begin{array}{l}
{ }^{A-2} Z+\Lambda+n \\
{ }^{A-2} Z+\Sigma^{-}+p
\end{array} \quad\right. \text { (HINMWD) }
$$

This kind of decay can only be investigated in the $D H$ systems. Some calculations [33,34] estimated the width of HINMWD of the order of 50 times smaller than the free $\Lambda$ decay width and therefore very high statistics will be required. As a positive aspect, the impulses of the nucleons and hyperons in final state lie in well definite ranges. For instance, in the ${ }_{\Lambda \Lambda}^{12} C$ hyper-nucleus, assuming $B_{\Lambda} \approx 11[\mathrm{MeV}]$ and neglecting $\Delta B_{\Lambda \Lambda}$, one gets: $400 \leq p_{\Lambda, n} \leq 433$ and $275 \leq p_{\Sigma, p} \leq 320$. The 
absence of one pion and the impulse short range could help to obtain good reconstruction efficiency in the experiments, partially compensating low statistics.

\section{The production of $\Lambda \Lambda$ hypernuclei}

To create a $\Lambda \Lambda$ and $\Xi$ hyper-nuclei it is necessary to insert $2 s$-quarks inside a nucleus. This can be achieved by transfer of strangeness from $K$-mesons to nucleons in the reactions (direct production):

$$
\begin{gathered}
K^{-}+{ }^{A} Z \rightarrow\left(\text { through: } \pi^{0}+{ }_{\Lambda}^{A}(Z-1)\right) \rightarrow K^{+}+{ }_{\Lambda \Lambda}^{A}(Z-2) \\
K^{-}+{ }^{A} Z \rightarrow K^{+}+{ }_{\Xi}^{A}(Z-1) \text { (through } \Xi \text { re-scattering in nucleus) }
\end{gathered}
$$

Both the above reactions require a two-step process inside the nucleus and the probability of the direct production is therefore very small. Measurements at AGS [35] reported an upper limit of 10 [nb/sr] for the forward differential cross section of Eq. (9) and a value of 90 [nb/sr] for Eq. (10), both on ${ }^{12} C$ targets.

A more efficient way is the so called indirect production, in which a hyperon is produced in a target nucleus through a (quasi free) 2-body reaction:

$$
K^{-}+{ }^{A} Z \rightarrow K^{+/ 0}+\Xi^{-}+\text {Residual Nucleus }(A-1)
$$

followed by the capture of the hyperon into another nucleus. The various steps of this process, which involve the $\Xi^{-}$deceleration to stop, the formation of a $\Xi^{-}$-atom and the absorption into the second nucleus, will be illustrated in the next Section. This production technique has been used at KEK and AGS to produce all the above reported data and is planned also in the future experiments at JPARC.

\section{The Double Hypernuclei in the PANDA experiment}

The PANDA Collaboration planned to investigate the $\Lambda \Lambda$ interaction inside the hyper-nucleus with the aim to collect a statistically considerable amount of data about the binding and separation energy. To fulfil this goal a totally new technique has been devised, based on the use of the intense antiproton beam available in the ring HESR at the future facility FAIR. The technique (2-target technique) consists in producing $\Xi^{-}$hyperons by antiprotons on a ${ }^{12} C$ primary target, located inside the beam pipe of HESR, in the reactions (quasi free in nucleus):

$$
\bar{p}+N \rightarrow \Xi^{+/ 0}+\Xi^{-}
$$

The use of a ring instead of an extracted beam makes the transmitted antiprotons again ready for passing through the target in the next round, thus increasing the effective intensity of the beam. 
The $\Xi^{-} \mathrm{s}$ are brought to rest in a secondary target, located outside the beam pipe, where they are captured into an atomic orbit, followed by absorption and conversion to $\Lambda \Lambda$ inside another nucleus. The steps of this process after the $\Xi^{+/ 0}, \Xi^{-}$pair creation are the following:

1) Strong slowing down of $\Xi^{-}$by re-scattering inside the residual nucleus and simultaneous $\Xi^{+/ 0}$ annihilation in the primary target, with production of at least 2 anti-kaons

2) Complete $\Xi^{-}$deceleration to rest inside the secondary target and capture into a $\Xi^{-}$atom

3) $\Xi^{-}$cascade through the atomic levels down to the absorption into a $\Xi^{-}$hyper-nucleus

4) Conversion reaction $\Xi^{-} p \rightarrow \Lambda \Lambda$ and (eventual) $\Lambda \Lambda$ sticking to the same residual nucleus or fragment

5) Decay of the $\Lambda \Lambda$ hyper-nucleus

Steps 1-5 are the same as in the $\Lambda \Lambda$ hyper-nuclei made by kaons: the different production efficiency is due to the available rates of projectiles and to the effects of the setup on the slowing down. It must be remarked that the separation into 2 targets allows to investigate different $\Lambda \Lambda$ hypernuclei maintaining the same (optimized) $\Xi^{-}$source. The main problems in bringing the $\Xi^{-} \mathrm{s}$ to rest is the decay along the path. The feasibility study of a suitable (even though not completely detailed) geometry, in the frame of the HESR machine, evaluated a rate $R_{\Xi} \approx 2.2 \cdot 10^{-3}$ of stopped to produced $\Xi^{-}$s, with a primary ${ }^{12} \mathrm{C}$ target [36]. From the scarce available data about the $D H$ one can roughly estimate that a fraction $R_{\Lambda \Lambda \Xi} \approx 5 \cdot 10^{-2}$ of stopped $\Xi^{-}$s become $\Lambda \Lambda$ hyper-nuclei. Therefore the performances of antiproton beam, primary target and detectors must be optimized keeping in mind this reduction $\left(\approx 10^{-4}\right)$ between the produced $\Xi^{-}$s per antiproton and the final number of $\Lambda \Lambda$ hypernuclei. The choice of the antiproton momentum was determined by the maximum cross section of Eq. (12), $\sigma_{\bar{p} p \rightarrow \Xi^{+} \Xi^{-}} \approx 2[\mu \mathrm{b}]$ at $3 \mathrm{GeV} / \mathrm{c}$ [37]. The time and space structure of the antiproton beam, together with the required performance of detectors, are discussed in the next Section.

\section{The Double Hypernuclei experimental setup}

The physics program of the PANDA experiment is concerning 3 main items: the charm spectroscopy, the double hyper-nuclei physics and the nucleon structure. The experimental apparatus has been designed in order to satisfy the needs of all the related measurements. It consists of 3 parts, the central detectors, the hyper-nuclear detectors and the forward detectors, which include a monitor of the beam luminosity. The setup shows a cylindrical symmetry around the beam axis. Details can be found in $[2,38]$. In the hyper-nuclear measurements the dedicated detectors ( $\gamma$ detectors, primary target, secondary target), lying in the forward region of the apparatus, will be used in addition to the central tracker (central region) and the luminosity monitor (forward region), as described in the following.

\section{$5.1 \gamma$ Detectors}

To detect unambiguously the $D H$ events it is mandatory to reconstruct all the particles in the final state. The $\gamma$ 's emitted in the conversion reaction play a major role in establishing the energy balance of the process. A system of 16 clusters, of 3 HPGe crystals each, has been designed to be located before the upstream pole of the solenoid of the apparatus, with an acceptance of approximately $1 / 3$ of the full solid angle. In this region the fringing field of the magnet ranges from 0.5 to 0.8 [T] and slightly modifies the resolution (typically around $2.5[\mathrm{KeV}]$ ) of the crystals [39]. After tests of 1 year duration, it has been observed that, for $\gamma$ 's energies between 0.08 and $1.34[\mathrm{MeV}]$, the resolution worsens less than $10 \%$ and the modification disappears when the field is switched off; moreover the 
efficiency remained constant along the whole duration of the tests [40]. Therefore the HPGe $\gamma$ detectors will be safely used in the PANDA experiment.

\subsection{Primary target}

The primary target has to be a nuclear solid target in order to allow the initial strong deceleration of the $\Xi^{-} \mathrm{s}$ and must be inserted inside an antiproton ring, HESR, described in [41]. The effect of this presence on the circulating antiproton bunch is a strong depletion due to the hadronic cross sections (nuclear scattering, annihilation...), which approximately increase as the power $2 / 3$ of the mass number of the nuclear target and to the Single Coulomb Scattering, which scales with the square of the atomic number [41]. The Touschek effect is independent and the straggling is weakly dependent (and not increasing with A) on the target. Moreover, if the annihilation rate is high, most part of the PANDA detectors become blind, blocking the data acquisition. These constraints suggested to build a wire-shaped, very thin $(\approx 3[\mu \mathrm{m}])$ primary target. A prototype has been already realized with special techniques (shaping with a pulsed Femto-LASER of 1064 [nm] wavelength) and submitted to some tests of mechanical and thermal resistance and radiation hardness. The positive results of the tests are reported in [42].

In spite of the reduced thickness, the particular time structure of the antiproton beam, which foresees a circulating bunch of $10^{10}$ antiprotons at the beginning of each cycle, produces an initial rate of annihilation not tolerable by the detectors, if the beam impinges centrally on the target. Profiting of the Gaussian shape of the transverse profile of the bunch, it has been suggested to overlap only the tail of the periphery of the cross section with the target and to slowly and continuously steer the beam toward the target position in the centre of the beam pipe. An important role in monitoring the steering of the beam will be played by the forward luminosity monitor. This technique is at present under study, together with the shape of the target frame and the system to extract the target during the beam preparation and re-insert it into the pipe [42]. Taking into account the ratios discussed in Section 4 and the expected performances of the HESR with the primary target inside, it has been estimated that a statistics of $\approx 100 \Lambda \Lambda$ hyper-nuclei detected in a single decay channel can be collected within $3 \div 4$ months of data taking.

\subsection{Secondary target}

The secondary target will consists of 3 modules, each one made of alternate layers of absorber (active target, in which $\Xi^{-}$stops and forms a $D H$ ) and Si- $\mu$ strips for tracking the charged particles generated in the $D H$ decay. The 3 parallelepiped shaped modules will be located around the beam pipe, covering 3 sides and leaving the forth free for the primary target displacement. The kaons emitted from the $\Xi^{+/ 0}$ annihilation in the primary target will be detected by the central tracker [38] and will be used to trigger the creation of the $\Xi^{-}-\Xi^{+/ 0}$ pair. Prototypes of the strips have been already realized and their read-out is now under test. Also some tests of the radiation hardness are planned, since this active/passive target should work very close to the interaction region, where the hadronic environment is very large. A final remark is concerning the flexibility in choosing the absorber. Several modules containing different absorbers can be mounted in different data taking, thus allowing to create several double hyper-nuclei and investigate their properties as a function of the mass number.

\section{$6 \Xi^{-}$atoms and $\Xi^{-}$hyper-nuclei at PANDA}

The goal of the first $D H$ data taking is the production and detection of a statistically significant amount of $\Lambda \Lambda$ hyper-nuclei and the measurement of their binding energy. The present activity of the 
Collaboration is dedicated to design, build and test the experimental devices, as well as to implement the simulation and reconstruction codes, in view of this goal. Nevertheless, as explained in Section 4, the $\Lambda \Lambda$ hyper-nuclei are only the final step of a process, in which also $\Xi^{-}$atoms and $\Xi^{-}$hyper-nuclei are necessarily formed. Therefore, if the setup can detect such strange systems, also the study of their properties can be planned.

Concerning the exotic $\Xi$ atoms, the hyperon cascade through the atomic levels emits $X$-rays in the range from several tens of $\mathrm{KeV}$ to few $\mathrm{MeV}$, depending on charge and mass of the atomic nucleus. The emission is isotropic and the HPGe array might perform spectroscopy measurements with high precision, perhaps enough to improve the accuracy of the present value of the $\Xi^{-}$mass. In the peripheral region of the nucleus, the shifts of the absorption levels depend on the nucleus and on the potential models used to perform the calculations. Some estimates, based on " $t \rho$ " potential, are reported in [43] for $\Xi^{-}-\mathrm{Cl}$ and show values of the shift slightly smaller than the HPGe resolution. Since other models (e.g. density-dependent potential) give higher values, measurements with suitable targets look not desperate, provided that enough time for high statistics will be assigned to the data taking. At present no effort is dedicated to the suitable simulation and reconstruction codes for $\Xi^{-}$ atoms, but it is not excluded for the future.

The situation for the $\Xi^{-}$hyper-nuclei looks different, because, even though the $\Xi^{-}$spectroscopy is based on the observation of emitted $\gamma$ 's in the expected range of few MeV, i.e. within the performance of the crystals, nevertheless the suppression due to the conversion reaction requires a high statistics, probably impossible to reach also at FAIR. Therefore, plans for such measurements are to be discarded.

\section{Conclusions}

The study of the $S=-2$ atoms and hyper-nuclei open a door toward the exploration of the nuclear interactions of multi-strange systems. Moreover, the $\Lambda \Lambda$ hyper-nuclei are the only tool to obtain data about the $s$-s quarks interaction. Many interesting aspects of the $S=-2$ physics, like the $\Xi^{-}$hyperonnucleus force, the nuclear levels of the $\Xi^{-}$hyper-nucleus, the $\Lambda \Lambda$ potential and the $\Lambda \Lambda$ weak decay, can be investigated in these systems and models of the interaction mechanisms can be tested. The status of art of the data at present is very poor and this is due to the experimental difficulty in producing such systems in large amount. The advent of the new facilities JPARC and FAIR, with their intense beams of kaons and antiprotons, seems open big perspectives to future. The PANDA Collaboration at FAIR aims to investigate the properties of the $\Lambda \Lambda$ hyper-nuclei getting a copious $D H$ production. The experiment takes advantage from the high rate of antiprotons stored in the HESR to produce a large rate of $\Xi^{-}-\Xi^{+/ 0}$ pairs inside the beam pipe of the ring: a suitable geometry will allow to stop a considerable fraction of the produced negative hyperons in a secondary target outside the pipe. The feasibility study of this technique has been performed with positive results. An important aspect is the possibility to get different $\Lambda \Lambda$ hyper-nuclei without modifying the rate of the $\Xi^{-}-\Xi^{+/ 0}$ pair production, but simply changing the stopping material in the secondary target. The problems related to this technique mainly concern the effects of the presence of the internal solid target inside the ring and the performances of the HPGe $\gamma$-detectors and of the active/passive secondary target. Prototypes of the primary target have been designed and already realized: the mechanical, thermal and radiation hardness tests gave positive results. A particular variable beam structure will be used to avoid the fast consuming of the antiproton bunch and the overwhelming background from the annihilations onto the detectors. The performances of the Germanium crystals inside a fringing field of $\approx 0.8[\mathrm{~T}]$ have been also tested with satisfying results: the resolution decreases less than $10 \%$ and the efficiency remains constant. After switching off the magnetic field, the crystals recover their original properties: this behaviour has been observed during long time operations, as it will be 
necessary in the long data taking. The design of the active part of the secondary target is completed and a prototype of the detector and read-out is under construction: the most important tests are concerning the radiation hardness, because of the large annihilation products where they are located and are planned in the early future. The activity on the simulation and reconstruction codes is in progress within the frame of the general PANDA software.

The hyper-nuclear setup of PANDA is suitable for investigating the $\Xi^{-}$atoms and $\Xi^{-}$hypernuclei spectroscopy too, without modifications of the experimental setup: in fact the $X$-rays and $\gamma$ rays emitted by these systems can be detected by HPGe array in the same data taking dedicated to the $\Lambda \Lambda$ hyper-nuclei. The difficulties for both these systems might arise from the very high statistics which is needed and, for the $\Xi^{-}$atoms, the required sharp resolution in detecting shift and width.

In conclusion, the PANDA project for the study of the $\Lambda \Lambda$ hyper-nuclei is in an advanced stage and promises a good improvement of the present collection of the experimental data.

\section{References}

1. M. Danysz et al., Nucl. Phys.49, 121 (1963)

2. The PANDA Collaboration, Technical Progress Report for PANDA: Strong Interaction Studies with Antiprotons, (2005).

3. T. Takahashi, Proc. of HYP2012, Barcelona, "Overview of the Hypernuclear program at JPARC

4. S. Lenz et al., Phys. Lett. B 41650 (1998)

5. G. Beer et al., Phys. Lett. B 53552 (2002)

6. F. Iazzi and K. Szymanska, Hyperfine Interactions,193, 89(2009)

7. Y. Yamamoto, Few-Body Syst. Suppl. 9, 145 (1995) (and ref. quoted therein)

8. C.Dover, A. Gal, Ann. Phys.146, 309 (1983)

9. T. Fukuda, Nucl. Phys.A639, 355c (1998)

10. I. Kumagai-Fuse, Y. Akaishi, Prog. Theor. Phys. 94, 151 (1995)

11. T. Motoba, Nucl. Phys.A691, 213c-219c (2001)

12. D. H. Wilkinson et al., Phys. Rev. Lett 3397 (1959)

13. J. Catala et al.,"Proc. of the International Conference on Hypernuclear Physics", Argonne, Illinois 758 (1969)

14. A.S. Mondal et al., Nuovo Cim. A54 333 (1979)

15. A. Bechdolff et al., Phys. Lett. B26 174 (1968).

16. C. B. Dover and A. Gal, Ann. Phys. 146309 (1983)

17. J.K. Ahn et al., Phys. Lett. B633 214-218 (2006)

18. S. Aoki et al., Nucl. Phys. A644 365 (1998)

19. P. Khaustov, et al., Phys. Rev. C61 054603 (2000)

20. W. Alberico, G. Garbarino, Phys. Rep. 369 1-109 (2002)

21. H. Takahashi et al., Phys. Rev. Lett. 87212502 (2001)

22. M. Danysz et al., Phys.Rev.Lett.11,29 (1963)

23. K. Nakazawa, in "The 20th Int. IUPAP Conf. on Few Body Problems in Physics", Fukuoka,(2012)

24. S. Aoki et al., Prog.Theor.Phys.85,1287(1991)

25. D. Prowse, Phys.Rev.Lett.17,782 (1966)

26. R.L. Jaffe, Phys. Rev. Lett. 38, 195 (1977)

27. S.R. Beane et al. (NPLQCD Collaboration), Phys. Rev. Lett. 106, 162001 (2011).

28. S.R. Beane et al. (NPLQCD Collaboration), Mod. Phys. Lett. A 26, 2587 (2011).

29. T. Inoue et al. (HALQCD Collaboration), Phys. Rev. Lett. 106, 162002 (2011).

30. K. Yamamoto et al., Phys. Lett. B478, 401-407 (2000) 
31. K. Itonaga, T. Ueda, T. Motoba, Nucl. Phys. A691, 197c (2001)

32. S.L. Olsen, "Properties of non-qqbar XYZ mesons and results of a search for the H-dibaryon", in "PoS of International Winter Meeting on Nuclear Physics,21-25 January 2013Bormio, Italy"

33. K. Nakazawa et al., Nucl. Phys. A639,345c-353c(1998)

34. A. Parrenos, A. Ramos, C. Bennhold, Phys. Rev. C65 015205 (2002)

35. M. May, Nucl. Phys. A 691, 226c (2001)

36. F. Ferro et al. Nucl.Phys. A789, 209 (2007)

37. B. Musgrave et al., Nuovo Cimento 35, 735 (1965)

38. W. Erni et al., Eur. Phys. J. A 49, 25 (2013)

39. A. Sanchez Lorente et al., Nucl. Inst. Meth. A573, 410-417 (2007)

40. K. Szymanska et. al. Nucl. Instr.and Meth A592, 486 (2008)

41. A. Lehrach et al., Nucl. Inst. Meth. A 561, 289 (2006)

42. H. Younis et al., J. Radioanal. Nucl. Chem. DOI 10.1007/s10967-013-2639-z

43. E. Friedman and A. Gal, Phys. Rep. 452,89 (2007) 